\title{
Korteweg-de Vries Equation; Asymptotic Behavior of Solutions
}

By

\author{
Shunichi TANAKA*
}

\section{\$. Introduction}

In [9] and [10], we have studied the initial value problem for the Korteweg-de Vries (KdV) equation

$$
u_{t}-6 u u_{x}+u_{x x x}=0
$$

by the inverse scattering method. In this paper we study the asymptotic behavior of the solutions as $t \rightarrow \pm \infty$.

Consider the Schrödinger equation

$$
-\phi^{\prime \prime}+u(x) \phi=\zeta^{2} \phi, \quad \zeta=\xi+i \eta
$$

over $(-\infty, \infty)$ with the potential $u(x)$ satisfying

$$
\int(1+|x|)|u(x)| d x<\infty
$$

(throughout the paper integration is taken over $(-\infty, \infty)$ unless otherwise indicated).

If $\operatorname{Im} \zeta \geqq 0$, there exist unique solution $f_{ \pm}(x, \zeta)$, of $(1.2)$, called the Jost solutions, which behave like $\exp ( \pm i \zeta x)$ as $x \rightarrow \pm \infty$. They are analytic in $\zeta, \operatorname{Im} \zeta>0$. If $\zeta=\xi$ is real, $f_{+}^{*}$, the complex conjugate of $f_{+}$, also satisfies (1.2) and the Wronskian $\left[f_{+}, f_{+}^{*}\right]$ is equal to $2 i \xi$. Therefore we have

$$
\begin{aligned}
& f_{-}=a(\xi) f_{+}^{*}+b(\xi) f_{+}, \\
& a(\xi)=(2 i \xi)^{-1}\left[f_{+}, f_{-}\right]
\end{aligned}
$$

Communicated by H. Yoshizawa, November 16, 1973.

* Department of Mathematics, Osaka University, Osaka. 


$$
b(\xi)=(2 i \xi)^{-1}\left[f_{-}, f_{+}^{*}\right] .
$$

By (1.4), $a(\xi)$ is the boundary value of the function $a(\zeta)$ which is analytic in $\operatorname{Im} \zeta>0$. The zeros of $\mathrm{a}(\zeta)$ in $\operatorname{Im} \zeta>0$ are purely imaginary (because the Jost solutions are linearly dependent and therefore square integrable at such $\zeta$ ) and simple. Their number is finite and we denote them by $i \eta_{1}, \ldots, i \eta_{N}$.

Function $r(\xi)=b(\xi) a(\xi)^{-1}$ is called the reflection coefficient and the positive numbers $c_{j}$ defined by

$$
c_{j}^{-1}=\int f_{+}\left(x, i \eta_{j}\right)^{2} d x
$$

are called the normalization coefficients. The collection $\left\{r(\xi), \eta_{j}, c_{j}\right\}$ is called the scattering data.

If $u(x)$ has rapidly decreasing derivatives, then $r(\xi)$ has also rapidly decreasing derivatives. Following Gardner, Greene, Kruskal and Miura (GGKM) [3], put

$$
\begin{aligned}
& r(\xi, t)=r(\xi) \exp \left(8 i \xi^{3} t\right), \quad-\infty<\xi<\infty, \\
& c_{j}(t)=c_{j} \exp \left(8 \eta_{j}^{3} t\right), \quad 1 \leqq j \leqq N .
\end{aligned}
$$

Then by the solvability of the inverse scattering problem, for each $t$ there exists a smooth potential $u(x, t)$ having $\left\{r(\xi, t), \eta_{j}, c_{j}(t)\right\}$ as its scattering data ([2], [10]). The function $u(x, t)$ satisfies the $\mathrm{KdV}$ equation ([7], [9], [10] ).

Let $u^{s}(x, t)$ be the potential whose scattering data is $\left\{0, \eta_{j}, c_{j}\right\}$, the reflectionless part of $u(x, t)$. The main result of this paper is formulated as

Theorem 1.1. Let $\varepsilon>0$ be arbitrarily fixed. As $t \rightarrow \pm \infty$

$$
\sup _{ \pm x> \pm \varepsilon t}\left|u(x, t)-u^{s}(x, t)\right| \longrightarrow 0 .
$$

Although the proof of Theorem 1.1 is independent of the fact that $u(x, t)$ satisfies (1.1), its interpretation is given in terms of particular solutions of (1.1) as follows.

The $\mathrm{KdV}$ equation is know to have travelling wave solutions of sppeed $c>0$ : 


$$
\begin{aligned}
& u(x, t)=s(x-c t-\delta ; c), \\
& s(x ; c)=-2^{-1} c \operatorname{sech}^{2}\left(2^{-1} c^{\frac{1}{2}} x\right) .
\end{aligned}
$$

Each of such solutions is called a solitary wave or soliton on account of its shape.

A significant role which reflectionless solutions $u^{s}(x, t)$ play in the theory of the $\mathrm{KdV}$ equation has been recognized by GGKM [3]. For $N=1, u^{s}(x, t)$ coincide with soliton solutions. In [3], several important observations have been made concerning the structure of the reflectionless solutions and in which sense the general solution of (1.1) is approximated by its reflectionless part.

The structure of reflectionless solutions is elementary and has been discussed by several authors. In particular the following asymptotic property of the reflectionless solutions has been established (see [8] for a proof).

Proposition 1.2. Assume that $\eta_{1}<\eta_{2}<\ldots<\eta_{N}$ and $p u t$

$$
\begin{aligned}
& \delta_{i}(j)=\eta_{j}^{-1} \log \left\{\left(\eta_{j}-\eta_{i}\right)\left(\eta_{j}+\eta_{i}\right)^{-1}\right\}, \\
& \delta_{j}^{ \pm}=\left(2 \eta_{j}\right)^{-1} \log c_{j}\left(2 \eta_{j}\right)^{-1}+\sum_{ \pm(i-j)>0} \delta_{i}(j) .
\end{aligned}
$$

Then

$$
u^{s}(x, t)-\sum_{j=1}^{N} s\left(x-4 \eta_{j}^{2} t-\delta_{j}^{ \pm} ; 4 \eta_{j}^{2}\right) \longrightarrow 0
$$

as $t \rightarrow \pm \infty$ uniformly in $x$.

Combination of Theorem 1.1 with Proposition 1.2 leads to

Theorem 1.3. As $t \rightarrow \pm \infty$

$$
\sup _{ \pm x> \pm \varepsilon t}\left|u(x, t)-\sum_{j=1}^{N} s\left(x-4 \eta_{j}^{2} t-\delta_{j}^{ \pm} ; 4 \eta_{j}^{2}\right)\right| \longrightarrow 0 .
$$

After the present work is completed, Shabat [7] has been published where a similar problem is discussed. The method employed in [7] is different from that of this paper.

In $\S 2$ we describe preliminary materials from inverse scattering theory which concern the Marchenko equation and the Gelfand-Levitan equation. Then Theorem 1.1 is proved in $\S 3$. 


\section{§. Integral Equations of the Inverse Problem.}

Most of the results formulated in this section are known. See also [1], [6] for generalities on the inverse scattering problem, in particular on the Marchenko equation and its solution.

For a function $\Omega$, in $L^{1}(a, \infty)$ for any $a$, define the integral operator $\Omega_{x}$ by

$$
\left(\Omega_{x} \varphi\right)(y)=\int_{0}^{\infty} \Omega(x+y+z) \varphi(z) d z
$$

as an operator in $L^{1}(0, \infty)$. By the Frechet-Kolmogorov theorem [1], p. 275], $\Omega_{x}$ is completely continuous.

Let $r(\xi)$ be a square-integrable continuous function which satisfies $|r(\xi)|<1$ for $\xi \neq 0, r^{*}(\xi)=r(-\xi)$. Let $\eta_{1}, \ldots, \eta_{N}$ be mutually different positive numbers and $c_{1}, \ldots, c_{N}$ be any positive numbers. Denote by $s=\left\{r(\xi), \eta_{j}, c_{j} ; j=1, \ldots, N\right\}$ the given data of the inverse problem. Put

$$
\Omega(x)=2 \sum_{j=1}^{N} c_{j} \exp \left(-2 \eta_{j} x\right)+\pi^{-1} \int r(\xi) \exp (2 i \xi x) d \xi
$$

By the argument in [2, p. 158-159], we have

Lemma 2.1. If $\Omega$ defined by (2.1) is bounded integrable in $(a, \infty)$ for any $a$, then

(a) the operator $I+\Omega_{x}$ has bounded inverse,

(b) $\left(I+\Omega_{x}\right)^{-1}$ is continuous in $x$ and bounded as $x \rightarrow \infty$ in the operator norm (topology).

Under the same assumption on $\Omega$ as in Lemma 2.1, there exists a unique solution $B(x, y)$, integrable in $y$, of the Marchnko equation

$$
B(x, y)+\int_{0}^{\infty} \Omega(x+y+z) B(x, z) d z+\Omega(x+y)=0 .
$$

The kernel $B$ is estimated as

$$
\int_{0}^{\infty}|B(x, y)| d y \leqq C(x)
$$

and

$$
|B(x, y)| \leqq C(x) \alpha(x+y)
$$


where $C(x)$ denotes a non-increasing function and

$$
\alpha(x)=\sup _{y \geq x}|\Omega(y)| .
$$

The differentiability of the kernel $B$ is based on

Lemma 2.2. Suppose that $\Omega$ defined by (2.1) is absolutely continuous and $\Omega, \Omega^{\prime}$ are in $L^{1}(a, \infty)$ for any $a$. Let $\psi_{x}$ be a vector in $L^{1}(0, \infty)$ depending differentiably on $x$ and let $\varphi_{x}$ be a solution of the operator equation

$$
\varphi_{x}+\Omega_{x} \varphi_{x}=\psi_{x}
$$

Then $\varphi_{x}$ is differentiable in $x$ and

$$
\varphi_{x}^{\prime}+\Omega_{x} \varphi_{x}^{\prime}=\psi_{x}^{\prime}-\left(\Omega^{\prime}\right)_{x} \varphi_{x}
$$

holds.

Proof. For the difference coefficient

$$
\varphi_{x, h}=h^{-1}\left(\varphi_{x+h}-\varphi_{x}\right)
$$

we have the equation

$$
\varphi_{x, h}+\Omega_{x} \varphi_{x, h}=\psi_{x, h}-h^{-1}\left(\Omega_{x+h}-\Omega_{x}\right) \varphi_{x+h} .
$$

The right hand side converges to $\psi_{x}^{\prime}-\Omega_{x}^{\prime} \varphi$ as $h \rightarrow 0$. By Lemma 2.1 (a), $\varphi_{x, h}$ converges as $h \rightarrow 0$ and the limit $\varphi_{x}^{\prime}$ satisfies (2.3) as asserted. Q.E.D.

This lemma leads to the differentiability of $B$ in the ordinary sense with respect to $x$ and to the estimates

$$
\begin{aligned}
& \int_{0}^{\infty}|(\partial / \partial x) B(x, y)| d y \leqq C(x), \\
& \mid(\partial / \partial x) B(x, y)+\Omega^{\prime}(x+y) ! \leqq C(x) \alpha(x+y)^{2} .
\end{aligned}
$$

Differentiating (2.2) with respect to $y$, the same estimates hold for the $y$-derivative.

Put

$$
f(x, \zeta)=\exp (i \zeta x)\left(1+\int_{0}^{\infty} B(x, y) \exp (2 i \zeta y) d y\right)
$$


and

$$
u(x)=-(\partial / \partial x) B(x, 0)
$$

If $r(\xi) \equiv 0$, then $f(x, \zeta)$ defined by (2.4) takes a simpler form (see [5]). In fact put

$$
a_{j k}(x)=c_{j}^{-1} \exp \left(2 \eta_{j} x\right) \delta_{j k}+\left(\eta_{j}+\eta_{k}\right)^{-1}
$$

$\left(\delta_{j k}\right.$ is Kroncker's delta). As the matrix $\left(a_{j k}\right)$ is nonsingular we denote by $h_{j}(x)$ the solution of the system of linear algebraic equations

$$
\sum_{k=1}^{N} a_{j k}(x) h_{k}(x)=-1
$$

Then

$$
f(x, \zeta)=\exp (i \zeta x)\left[1+i \sum_{j=1}^{N} h_{j}(x)\left(\zeta+i \eta_{j}\right)^{-1}\right]
$$

holds.

Denoting by $\left(b_{j k}\right)$ the matrix which is inverse to $\left(a_{j k}\right)$, we have obvious expressions

$$
\begin{aligned}
& h_{j}(x)=-\sum_{k=1}^{N} b_{j k}(x), \\
& h_{j}^{\prime}(x)=-2 \sum_{k=1}^{N} b_{j k}(x) \eta_{k} c_{k}^{-1} \exp \left(2 \eta_{k} x\right) h_{k}(x) .
\end{aligned}
$$

In the following we consider the data with the properties that $\Omega$ is twice differentiable and $\Omega, \Omega^{\prime}, \Omega^{\prime \prime}$ are in $L^{1}(a, \infty)$ for any $a$. Then $B$ is twice differentiable with its derivatives integrable in $y$ in $(0, \infty)$. The function $f$ is twice differentiable and

$$
-f^{\prime \prime}+u f=\zeta^{2} f
$$

holds.

Rewrite (2.4) as

$$
\begin{aligned}
& f(x, \zeta)=\exp (i \zeta x)+\int_{x}^{\infty} A(x, y) \exp (i \zeta y) d y, \\
& A(x, y)=2^{-1} B\left(x, 2^{-1}(y-x)\right) .
\end{aligned}
$$


By an analogous argument to that given by Gasimov [4, Lemma 3.5.2], it is easy to show

Lemma 2.3. Put

(2.11) $\quad A_{-}(x, y)=2^{-1} \Omega\left(2^{-1}(x+y)\right)+2^{-1} \int_{y}^{\infty} A(y, z) \Omega\left(2^{-1}(x+z)\right) d z, \quad x<y$.

Then

$$
\exp (i \zeta x)=f(x, \zeta)+\int_{x}^{\infty} A_{-}(x, y) f(y, \zeta) d y
$$

holds.

Combining (2.9) and (2.12) we get an equality

$$
A(x, y)+A_{-}(x, y)+\int_{x}^{y} A(x, z) A_{-}(z, y) d z=0 \text {. }
$$

Put

$$
\begin{aligned}
& \varphi_{f}(\xi)=\int \varphi(x) f(x, \xi) d x, \\
& \varphi_{j}=\int \varphi(x) f(x, i \eta) d x, \quad j=1, \ldots, N .
\end{aligned}
$$

We derive an inversion formula which reproduces $\varphi$ from $\varphi_{f}$ and $\varphi_{j}$.

Define the operators $A_{ \pm}$by

$$
\begin{aligned}
& \left(A_{+} \varphi\right)(x)=\int_{-\infty}^{x} A(y, x) \varphi(y) d y \\
& \left(A_{-} \varphi\right)(x)=\int_{x}^{\infty} A-(x, y) \varphi(y) d y .
\end{aligned}
$$

Lemma 2.4. Suppose that $\varphi$ is 0 for $x<a$, is in $L^{1} \cap L^{2}$ and is absolutely continuous with derivative also in $L^{1} \cap L^{2}$. Then $\varphi_{f}(\xi)$ is integrable.

Proof. By the estimates for the kernel $B$ and its derivatives, $A_{+} \varphi$ and $\left(A_{+} \varphi\right)^{\prime}$ are bounded integrable functions. As $\varphi_{f}$ is the Fourier transform of $\left(I+A_{+}\right) \varphi,\left(1+\xi^{2}\right)^{\frac{1}{2}} \varphi_{f}(\xi)$ is square integrable. By Schwarz' inequality $\varphi_{f}$ is integrable.

Q.E.D. 
Theorem 2.5 (Inversion formula). If $\varphi$ satisfies the condition of Lemma 2.4, then we have

$$
\begin{aligned}
\varphi(x) & =(2 \pi)^{-1} \int[f(x,-\xi)+r(\xi) f(x, \xi)] \varphi_{f}(\xi) d \xi \\
& +\sum_{j=1}^{N} c_{j} \varphi_{j} f\left(x, i \eta_{j}\right),
\end{aligned}
$$

the right hand side converging absolutely.

Proof. The absolute convergence of the right hand side is a consequence of Lemma 2.4.

By (2.11) and the Marchenko equation, we have

$$
\begin{aligned}
\left(A_{+} \varphi\right)(x)= & \left(A_{-} \varphi\right)(x)-2^{-1} \int\left[\Omega\left(2^{-1}(x+z)\right)\right. \\
& \left.+\int_{y}^{\infty} \Omega\left(2^{-1}(x+z)\right) A(y, z) d z\right] \varphi(y) d y
\end{aligned}
$$

Substitution of $\Omega$ by (2.1) then yields

$$
\begin{aligned}
\left(A_{+} \varphi\right)(x)= & \left(A_{-} \varphi\right)(x)-(2 \pi)^{-1} \int \varphi_{f}(\xi) r(\xi) \exp (i \xi x) d \xi \\
& -\sum_{j=1}^{N} c_{j} \varphi_{f}\left(i \eta_{j}\right) \exp \left(-\eta_{j} x\right) .
\end{aligned}
$$

Take a function $\psi$ in class $C^{1}$ with compact support. Denoting by $\langle$,$\rangle the inner product for L^{2}(-\infty, \infty)$ and putting

$$
I=(2 \pi)^{-1}\left\langle\varphi_{f}, \psi_{f}\right\rangle
$$

we have

$$
\begin{aligned}
I= & \left\langle\left(I+A_{+}\right) \varphi,\left(I+A_{+}\right) \psi\right\rangle \\
= & \left\langle\left(\left(I+A_{-}\right) \varphi,\left(I+A_{+}\right) \psi\right\rangle\right. \\
& -(2 \pi)^{-1} \int \varphi_{f}(\xi) \psi_{f}^{*}(\xi) r(\xi) d \xi-\sum_{j=1}^{N} c_{j} \varphi_{f}\left(i \eta_{j}\right) \psi_{f}^{*}\left(i \eta_{j}\right) .
\end{aligned}
$$

The inner product term in the last expression for $I$ being equal to $\langle\varphi, \psi\rangle$ by (2.13), the assertion follows by the arbitrariness of $\psi$.

Q.E.D.

Next also consider given data $s^{\circ}$ along with $s$ and put the superfix ${ }^{\circ}$ to quantities associated with $s^{\circ}$. Lemma 2.3 now makes it possible to 
define the transformation kernel $K$ between $f(x, \zeta)$ and $f^{\circ}(x, \zeta)$ in the following sense.

Put

$$
K(x, y)=A(x, y)+A_{-}^{\circ}(x, y)+\int_{x}^{y} A(x, z) A_{-}^{\circ}(z, y) d z
$$

Then

$$
f(x, \zeta)=f^{\circ}(x, \zeta)+\int_{x}^{\infty} K(x, y) f^{\circ}(y, \zeta) d y
$$

holds. The difference of $u$ and $u^{\circ}$ is reproduced by

$$
u(x)-u^{\circ}(x)=-d / d x K(x, x) .
$$

We have also

$$
f^{\circ}(x, \zeta)=f(x, \zeta)+\int_{x}^{\infty} K_{-}(x, y) f(y, \zeta) d y
$$

with the kernel $K$-defined in an obvious way.

Theorem 2.6. If $r$ and $r^{\circ}$ are supposed to be integrable in addition to the assumptions already made on $s$ and $s^{\circ}$, the Gelfand-Levitan equation for the kernel $K$ holds:

$$
\begin{gathered}
K(x, y)+\Omega(x, y)+\int_{x}^{\infty} \Omega(y, z) K(x, z) d z=0, \quad x<y \\
\begin{aligned}
& \Omega(x, y)=F(x, y)+\sum_{j=1}^{N} c_{j} f^{\circ}\left(x, i \eta_{j}\right) f^{\circ}\left(y, i \eta_{j}\right) \\
&-\sum_{k=1}^{N^{\circ}} c_{k}^{\circ} f^{\circ}\left(x, i \eta_{k}^{\circ}\right) f^{\circ}\left(y, i \eta_{k}^{\circ}\right), \\
& F(x, y)=(2 \pi)^{-1} \int\left(r(\xi)-r^{\circ}(\xi)\right) f^{\circ}(x, \xi) f^{\circ}(y, \xi) d \xi
\end{aligned}
\end{gathered}
$$

Proof. We follow $[1, \S 8]$ where an analogous derivation is given. For a function $\varphi$ in class $C^{2}$ with compact support, we have

$$
\varphi_{f^{\circ}}=(\varphi+\psi)_{f}
$$

with

$$
\psi(x)=\int_{-\infty}^{x} K_{-}(y, x) \varphi(y) d y
$$


Supposing that the support of $\varphi$ is contained in $[a, b]$ and applying the inversion formula for the data $s$ to $\varphi+\psi$, we have

$$
\begin{gathered}
(2 \pi)^{-1} \int[f(x,-\xi)+r(\xi) f(x, \xi)] \varphi_{f^{\circ}}(\xi) d \xi \\
+\sum_{k=1}^{N} c_{k} f\left(x, i \eta_{k}\right) \varphi_{f^{\circ}}\left(i \eta_{k}\right)=0, \quad x<a .
\end{gathered}
$$

Put (2.17) into this relation and again use the inversion formula for the data $s^{\circ}$. Rearranging the terms, we get (2.18) by the arbitrariness of $\varphi$.

Q.E.D.

\section{§3. Proof of Theorem 1.1}

Let a potential $u(x)$ be given and $\left\{r(\xi), \eta_{j}, c_{j}\right\}$ be its scattering data. Consider the data $s(t)=\left\{r(\xi, t), \eta_{j}, c_{j}(t)\right\}$ depending parametrically on $t, r(\xi, t)$ and $c_{j}(t)$ determined by (1.6) and (1.7).

Lemma 3.1. If $u(x)$ is six times differentiable with rapidly decreasing derivatives, then $r(\xi)$ is $C^{\infty}$ and $\xi^{7} r^{(i)}(\xi)(i=0,1,2)$ are bounded.

Proof. As the detailed proof has been given in [10], we only indicate the outline. The Jost solutions are represented as

$$
f_{ \pm}(x, \zeta)=\exp (i \zeta x)\left(1 \pm \int_{0}^{ \pm \infty} B_{ \pm}(x, y) \exp (2 i \zeta y) d y\right)
$$

the kernels $B_{ \pm}$satisfying certain integral equations (see [2, p. 142]. Insertion of the expressions into (1.4), (1.5) leads to

$$
\begin{aligned}
& 2 i \xi(a(\xi)-1)=-\int u(y) d y+\int_{0}^{\infty} \Gamma(y) \exp (2 i \xi y) d y \\
& 2 i \xi b(\xi)=\int \Pi(y) \exp (-2 i \xi y) d y
\end{aligned}
$$

$\Gamma$ and $\Pi$ being integrable functions expressed by $B_{ \pm}$and their derivatives. The analytical properties of $a, b$ and then $r$ are deduced from the above formulas.

Q.E.D.

We can now apply the Gelfand-Levitan equation (2.18) to data $s(t)$ 
and $s^{\circ}(t)=\left\{0, \eta_{j}, c_{j}(t)\right\}$. We understand that the additional parameter $t$ appearing in the quantities in (2.18) indicate that they correspond to data $s(t)$.

Putting $B(x, y ; t)=2 K(x, x+2 y ; t)$, we rewrite $(2.18)$ as

$$
\begin{aligned}
& B(x, y ; t)+2 F(x, x+2 y ; t)+2 \int_{0}^{\infty} F(x+2 z, x+2 y ; t) B(x, z ; t) d z=0, \\
& F(x, y ; t)=(2 \pi)^{-1} \int r(\xi, t) f^{\circ}(x, \xi ; t) f^{\circ}(y, \xi ; t) d \xi
\end{aligned}
$$

For an integrable function $g$, we study certain asymptotic properties of the integral

$$
G(x, t)=\int g(\xi) \exp \left(8 i \xi^{3} t+2 i \xi x\right) d \xi
$$

in $\{x>\varepsilon t, t>0\}, \varepsilon>0$ being fixed arbitrarily.

Lemma 3.2. If $g(\xi)$ is bounded and absolutely continuous with the bounded derivative, then $t G(x, t)$ is bounded in $\{x>\varepsilon t, t>0\}$. If moreover the second derivative of $g(\xi)$ exists and is bounded, $t^{2} G(x, t)$ is also bounded.

Proof. Integration by part leads to

$$
\begin{aligned}
G(x, t)=i \int[ & \left.g^{\prime}(\xi)\left(24 \xi^{2} t+2 x\right)^{-1}-g(\xi) 24 \xi t\left(24 \xi^{2} t+2 x\right)^{-2}\right] \\
& \times \exp \left(8 i \xi^{3} t+2 i \xi x\right) d \xi
\end{aligned}
$$

so that $t G(x, t)$ is bounded. Again integrating by parts, we obtain the second assertion.

Q.E.D.

Lemma 3.3. Suppose that $g(\xi) \xi^{4}$ is bounded integrable and $g^{\prime}(\xi) \xi^{4}$, $g^{\prime \prime}(\xi) \xi^{4}$ are bounded. Then $x^{2} G(x, t)$ is bounded in $\{x>\varepsilon t, t>0\}$.

Proof. Performing integration by parts twice, we have

$$
x^{2} G(x, t)=-\int\left(d^{2} / d \xi\right)^{2}\left(g(\xi) \exp \left(8 i \xi^{3} t\right)\right) \exp (2 i \xi x) d \xi
$$

By Lemma 3.2 the assertion is proved.

Q.E.D.

Next we consider the quantities introduced in relation to (2.6). 
Lemma 3.4. $\quad h_{j}(x, t)$ and $h_{j}^{\prime}(x, t)$ are bounded.

Proof. We have

$$
\operatorname{det}\left(a_{j k}(x, t)\right)=\Sigma \alpha\left(\varepsilon_{1}, \ldots, \varepsilon_{N}\right) \prod_{j=1}^{N} \exp \left\{\varepsilon_{j} 2 \eta_{j}\left(x-4 \eta_{j}^{2} t\right)\right\}
$$

where the sum is taken over all possible choice of $\varepsilon_{j}=0$ or 1 . By the same argument as is given in [3, Lemma 2], $a\left(\varepsilon_{1}, \ldots, \varepsilon_{N}\right)>0$. So we have

$$
\left|b_{j k}(x, t)\right| \leqq C\left\{1+\exp \left(2 \eta_{k}\left(x-4 \eta_{k}^{2} t\right)\right\}^{-1} .\right.
$$

By (2.7) and (2.8), the lemma follows.

Q.E.D.

After these preparations, we proceed to the study of the GelfandLevitan equation.

Lemma 3.5. Let $H(x, y, z ; t)$ denote one of $F(x+2 z, x+2 y ; t)$, $(\partial / \partial x) F(x+2 z, x+2 y ; t)$ and $(\partial / \partial y) F(x+2 z, x+2 y ; t)$. Then $(x+y+z)^{2}$ $H(x, y, z ; t)$ is bounded in $\{x>\varepsilon t, t>0, y, z \geqq 0\}$.

Proof. Using (2.6), we can write $H$ into the form

$$
\begin{aligned}
& H(x, y, z ; y t)=\sum_{j=1}^{N} a_{j}(x, y, z ; t) G_{j}(x+y+z ; t), \\
& G_{j}(x, t)=\int g_{j 1}(\xi) \exp \left(8 i \xi^{3} t+2 i \xi x\right) d \xi
\end{aligned}
$$

with bounded coefficients $a_{j}$ and functions $g_{j}(\xi)$ satisfying the conditions of Lemma 3.2.

Q.E.D.

Lemma 3.6. $\int_{0}^{\infty}|B(x, y ; t)| d y$ and $\int_{0}^{\infty}|\partial / \partial x B(x, y ; t)| d y$ are bounded in $D_{a, \varepsilon}=\{(x, t) ; x>a, x>\varepsilon t, t>0\}$ for certain $a$.

Proof. Define

$$
T_{x, t} \varphi(y)=\int_{0}^{\infty} F(x+2 z, x+2 y ; t) \varphi(z) d z
$$

as an operator on $L^{1}(0, \infty)$. By the previous lemma we can take $a>0$ so large that $\left\|T_{x, t}\right\|<2^{-1}-\delta$ in $D_{a, \varepsilon}$. Solving (3.1) in $L^{1}(0, \infty)$ by iteration, we have 


$$
\int_{0}^{\infty}|B(x, y ; t)| d y \leqq C \int_{0}^{\infty}|F(x, x+2 y ; t)| d y
$$

in $D_{a, \varepsilon}$. Again by the previous lemma the first assertion follows.

Applying the same argument to

$$
\begin{aligned}
(\partial / \partial x) & B(x, y ; t)+2 \int_{0}^{\infty} F(x+2 z, x+2 y ; t)(\partial / \partial x) B(x, z ; t) d z \\
= & -2(\partial / \partial x+\partial / \partial y) F(x, x+2 y ; t) \\
& -2 \int_{0}^{\infty}(\partial / \partial x+\partial / \partial y) F(x+2 z, x+2 y ; t) B(x, z ; t) d z
\end{aligned}
$$

the second assertion follows.

Q.E.U.

Now we conclude that by (3.3), Lemmas 3.4 and 3.5 that $\sup _{x>\varepsilon t, y \geqq 0}$ $|\partial / \partial x B(x, y ; t)|$ tends to zero as $t \rightarrow \infty$. Putting $y=0$, we have proved the part of Theorem 1.1 which concerns the limit for $t \rightarrow \infty$. The other part can be handled similarly.

\section{References}

[1] Faddeev, L. D., The inverse problem in the quantum theory of scattering, J. Maih. Phys. 4 (1963), 72-104.

[2] Faddeev, L. D., Properties of the S-matrix of the one-dimensional Schrödinger equation, Amer. Math. Soc. Translations (2) 65 (1967), 139-166 [Russian original Proc. Steklov Inst. Math. 73 (1965), 314-336].

[ 31 Gardner, O. S., Greene, J. M., Kruska!, M. D. and Miura, R. M., Method for solving the Korteweg-de Vries equations, Phys. Rev. Letters, 19 (1967), 1095-1097.

[4] Gasimov, M. G., Inverse problem of scattering for Dirac system of order 2n, Trudy Moskov Obsc. 19 (1968), 41-112 (Russian).

[5] Kay, I. and Moses, H. E., Reflectionless transmission through dielectrics and scattering potentials, J. Appl. Phys. 27 (1956), 1503-1508.

[6] Marchenko, V. A., Spectral theory of Sturm-Liouville operators, Kiev, 1972 (Russian).

[ 7 ] Shabat, A. B., On Korteweg-de Vries equation, Dokl. Akad. Nauk SSSR 211 (1973), 13101313 (Russian).

[8] Tanaka, S., On the N-tuple wave solutions of the Korteweg-de Vries equation. Publ. R.I.M.S. Kyoto Univ. 8 (1973), 419-427.

[9] Tanaka, S., Analogue of Fourier's method for Korteweg-de Vries equation, Proc. Japan Acad. 48 (1972), 647-650.

[10] Tanaka, S., Korteweg-de Vries equation: construction of solutions in terms of scattering data, Osaka J. Math. 11 (1974), 49-59.

[11] Yosida, K., Functional Analysis, Springer-Verlag, Berlin-Göttingen-Heidelberg, 1965. 
